\title{
Electronic and Magnetic Properties of $\mathrm{Co}_{3} \mathrm{~V}_{2} \mathrm{O}_{8}$ Compound
}

\author{
A. JEZIERSKI ${ }^{a}$, R. SzYMCZAK ${ }^{b}$, H. SzYMCZAK ${ }^{b}$ AND J. KACZKOWski ${ }^{a}$ \\ ${ }^{a}$ Institute of Molecular Physics, Polish Academy of Sciences \\ M. Smoluchowskiego 17, 60-179 Poznań, Poland \\ ${ }^{b}$ Institute of Physics, Polish Academy of Sciences \\ al. Lotników 32/46 02-668 Warszawa, Poland
}

\begin{abstract}
The band structures and the magnetic properties of $\mathrm{Co}_{3} \mathrm{~V}_{2} \mathrm{O}_{8}$ compound have been calculated by ab initio scalar-relativistic full potential local orbital method within the local spin density approximation. The band calculations were performed for para-, ferro-, and antiferromagnetic $\mathrm{Co}_{3} \mathrm{~V}_{2} \mathrm{O}_{8}$ compounds. The main contribution to the density of states at the Fermi energy comes from minority $3 d$ states of cobalt atoms.
\end{abstract}

PACS numbers: 71.20.-b, 71.15.-m, 71.15.Ap, 71.15.Mb, 71.20.Be

\section{Introduction}

The electronic and magnetic properties of $\mathrm{TM}_{3} \mathrm{~V}_{2} \mathrm{O}_{8}$ (where $\mathrm{TM}=\mathrm{Ni}, \mathrm{Co}, \mathrm{V}$ ) compounds have been studied in the last years [1-6]. These systems crystallise into orthogonal (space group Cmca, No. 64) type structure. The $\mathrm{TM}_{3} \mathrm{~V}_{2} \mathrm{O}_{8}$ compounds are highly frustrated because of specific geometry and they form new variants of kagome lattice [3-6]. In this work we report the electronic structure and magnetic properties of $\mathrm{Co}_{3} \mathrm{~V}_{2} \mathrm{O}_{8}$ compound calculated by $a b$ initio scalar-relativistic full potential local orbital (FPLO 7.00) method [7-11]. The outline of the method is presented in Sect. 2, and the results are given in Sect. 3.

\section{Method of calculations}

The band calculations were performed for the experimental lattice parameters [2] for the $\mathrm{Co}_{6} \mathrm{~V}_{4} \mathrm{O}_{16}$ compound. We assumed $a=6.034 \AA, b=11.488 \AA$, and $c=8.300 \AA$ [2]. The unit cell consisted of 26 atoms: six cobalt atoms (four $\mathrm{Co} 1$ and two $\mathrm{Co} 2$ ), four vanadium atoms and sixteen oxygen atoms (four $\mathrm{O} 1$, four O2, and eight O3). The electronic densities of states (DOS) and the magnetic properties were calculated by using the scalar-relativistic FPLO-7 method within the density functional theory (DFT) [7-11]. The exchange-correlation potential was taken in the form proposed by Perdew and Wang [12]. The scalar-relativistic self-consistent calculations were performed for $301 k$-points in the irreducible Brillouin zone. We have performed the band calculations for para-, ferro- and antiferromagnetic structures.

\section{Results and discussion}

The total electronic DOS for paramagnetic $\mathrm{Co}_{6} \mathrm{~V}_{4} \mathrm{O}_{16}$ is presented in Fig. 1. The Fermi level is located into

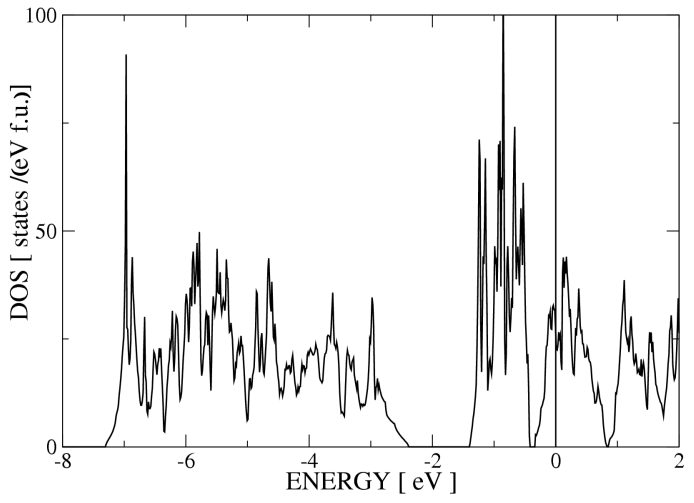

Fig. 1. Total density of states of the paramagnetic $\mathrm{Co}_{6} \mathrm{~V}_{4} \mathrm{O}_{16}$ compound.

valley between two peaks. The band structure near the Fermi level is plotted in Fig. 2. We observe the indirect gap between $Y-Z-T$ directions. The total density of states for ferromagnetic $\mathrm{Co}_{6} \mathrm{~V}_{4} \mathrm{O}_{16}$ compound is plotted in Fig. 3. The partial densities of states in Fig. 4 present the contributions from $\mathrm{Co} 1, \mathrm{Co} 2, \mathrm{~V}, \mathrm{O} 1, \mathrm{O} 2$ and $\mathrm{O} 3$ atoms to the total DOS. The main contribution to the density of states at the Fermi level $N\left(E_{\mathrm{F}}\right)$ comes from $3 d$ states of Co1 and Co2 atoms. The contributions from vanadium and oxygen atoms are small. The calculated total magnetic moment is $8.73 \mu_{\mathrm{B}}$ per $\mathrm{Co}_{3} \mathrm{~V}_{2} \mathrm{O}_{8}$. The local magnetic moments on Co sites are 2.45 and $2.46 \mu_{\mathrm{B}}$, respectively. We observe small magnetic moment on vanadium $\left(m=0.19 \mu_{\mathrm{B}}\right)$ and on oxygen atoms $\left(m=0.15,0.14\right.$ and $\left.0.10 \mu_{\mathrm{B}}\right)$, respectively. The density of states at the Fermi energy is $N\left(E_{\mathrm{F}}\right)=26.68$ (states $/(\mathrm{eV}$ f.u. $)$ ) for paramagnetic and $N\left(E_{\mathrm{F}}\right)=28.75$ (states $/(\mathrm{eV}$ f.u. $)$ ) for ferromagnetic $\mathrm{Co}_{6} \mathrm{~V}_{4} \mathrm{O}_{16}$. In the unit cell Co atoms occupy two positions. In the calculation for the ferromagnetic phase we 


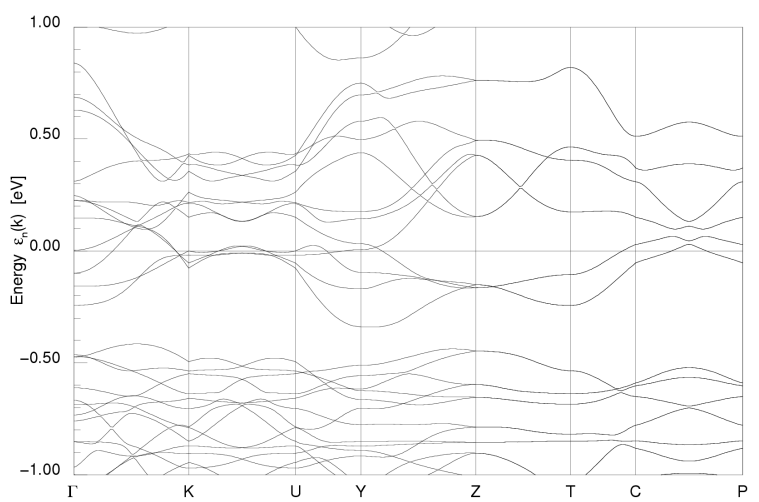

Fig. 2. Band structure of the paramagnetic $\mathrm{Co}_{6} \mathrm{~V}_{4} \mathrm{O}_{16}$ compound.

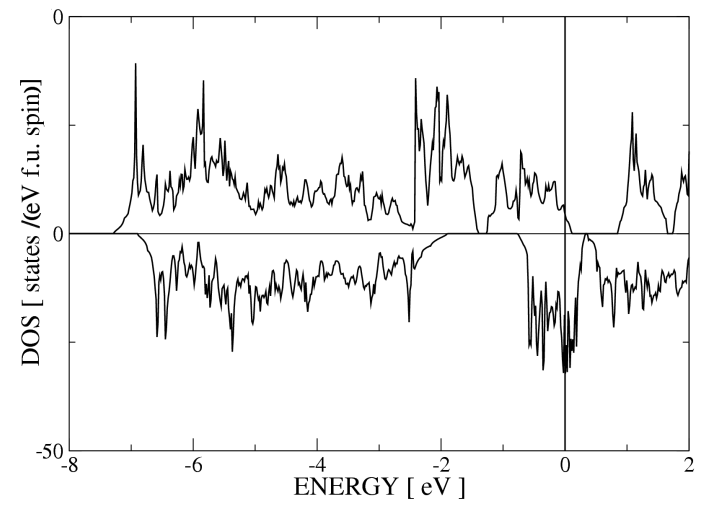

Fig. 3. Total density of states of the ferromagnetic $\mathrm{Co}_{6} \mathrm{~V}_{4} \mathrm{O}_{16}$ compound.

assumed that the magnetic moments had the same direction on each Co site. In the case of antiferomagnetic ordering of cobalt, we put magnetic moment up on Co1 $(0.25,0.1312,0.25)$ and down on Co2 $(0,0,0)$. For such configuration we have obtained the total magnetic moment $m=3.14 \mu_{\mathrm{B}}$ per $\mathrm{Co}_{3} \mathrm{~V}_{2} \mathrm{O}_{8}$. The local magnetic mo-

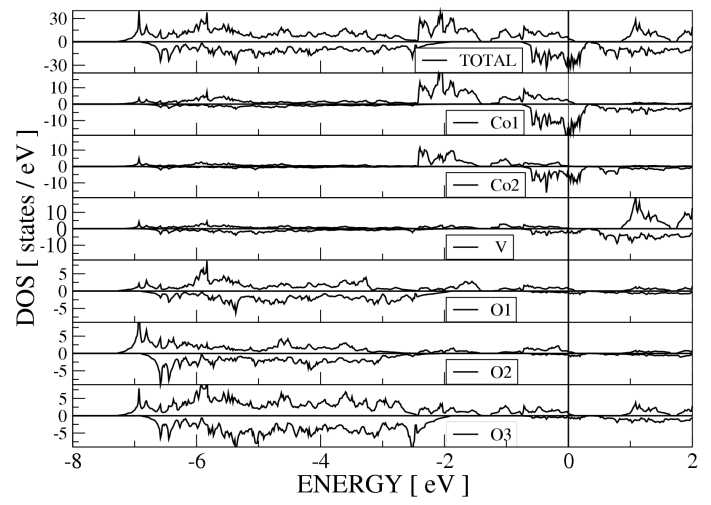

Fig. 4. Total and local density of states of the ferromagnetic $\mathrm{Co}_{6} \mathrm{~V}_{4} \mathrm{O}_{16}$ compound.

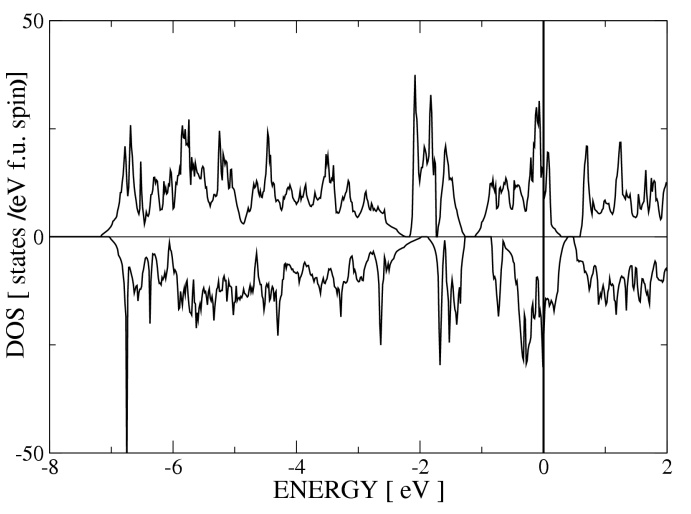

Fig. 5. Total density of states of the antiferromagnetic $\mathrm{Co}_{6} \mathrm{~V}_{4} \mathrm{O}_{16}$ compound.

ments on cobalt, vanadium and oxygen atoms assumed the following values: $m_{\mathrm{Co} 1}=2.29 \mu_{\mathrm{B}}, m_{\mathrm{Co} 2}=-1.87 \mu_{\mathrm{B}}$, $m_{\mathrm{V}}=-0.02 \mu_{\mathrm{B}}, m_{\mathrm{O}(1,2,3)}=0.13,0.02,0.04 \mu_{\mathrm{B}}$, respectively. The total density of states for $\mathrm{Co}_{6} \mathrm{~V}_{4} \mathrm{O}_{16}$ compound in antiferromagnetic ordering (according to definition presented above) is presented in Fig. 5. The density of states at the Fermi energy increased up to $N\left(E_{\mathrm{F}}\right)=41.05$ (states $/(\mathrm{eV}$ f.u. $\left.)\right)$. The scalar-relativistic FPLO calculations indicate that the total energy of ferromagnetic and antiferromagnetic structures is lower than for paramagnetic phase. However, the difference of the total energy between ferromagnetic and antiferromagnetic phase is small (0.007 Ry).

\section{Conclusions}

In this paper we have presented electronic and magnetic properties of $\mathrm{Co}_{3} \mathrm{~V}_{2} \mathrm{O}_{8}$ compound. The scalar-relativistic full-potential FPLO calculations have shown that the difference between the total energy of ferromagnetic and antiferromagnetic phase was small. We observed that main contributions to the density of states near the Fermi level came from $3 d$ states of cobalt atoms.

\section{Acknowledgments}

Work financed from the science resources as a joint research program within scientific network "Materials with strongly correlated electrons: manufacturing, research and applications" (MSSE).

\section{References}

[1] D. Aoki, Y. Katayama, R. Settai, Y. Inada, Y. Onuki, H. Harima, Z. Kletowski, Acta Crystallogr. B 29, 2304 (1973).

[2] R. Szymczak, M. Baran, R. Diduszko, J. Fink-Finowicki, M. Gutowska, A. Szewczyk, H. Szymczak, Phys. Rev. B 73, 094425 (2006).

[3] H. Szymczak, R. Szymczak, M. Baran, J. Fink-Finowicki, B. Krzymanska, P. Aleshkevych, A. Jezierski, Acta Phys. Pol. A 114, 35 (2008). 
[4] Y. Chen, J.W. Lynn, Q. Huang, F.M. Woodward, T. Yildrim, G. Lawes, A.P. Ramirez, N. Rogado, R.J. Cava, A. Aharony, O. Entin-Wohlman, A.B. Harris, Phys. Rev. B 74, 014430 (2006).

[5] M. Kenzelmann, A.B. Haris, A. Aharony, O. EntinWohlman, T. Yildrim, Q. Huang, S. Park, G. Lawes, C. Broholm, N. Rogado, R.J. Cava, K.H. Kim, G. Jorge, A.P. Ramirez, Phys. Rev. B 74, 014429 (2006).

[6] E. Morosan, J. Fleitman, T. Klimczuk, R.J. Cava, Phys. Rev. B 76, 144403 (2007).

[7] H. Eschrig, The Fundamentals of Density Functional Theory, 2nd ed., Edition Gutenbergplatz, Leipzig 2003.
[8] K. Koepernik, H. Eschrig, Phys. Rev. B 59, 1743 (1999).

[9] K. Koepernik, B. Velicky, R. Hayn, H. Eschrig, Phys. Rev. B 55, 5717 (1997).

[10] I. Opahle, K. Koepernik, H. Eschrig, Phys. Rev. B 60, 14035 (1999)

[11] FPLO code http://www.fplo.de.

[12] J.P. Perdew, Y. Wang, Phys. Rev. B 45, 13244 (1992). 